Research Article

\title{
Comprehensive Ahead Prospecting of Tunnels in Severely Weathered Rock Mass Environments with High Water Inrush Risk: A Case Study in Shaanxi Province
}

\author{
Lichao Nie, ${ }^{1}$ Yongheng Zhang $\mathbb{D}^{1},{ }^{1}$ Maoxin Su, ${ }^{1}$ Yijun Geng, ${ }^{2}$ Zhengyu Liu $\mathbb{D}^{1},{ }^{1}$ Kerui Fan, \\ Bing Yan, ${ }^{1}$ and Junfeng Shen ${ }^{1}$ \\ ${ }^{1}$ Shandong University, Geotechnical \& Structural Engineering Research Centre, Jinan 250100, China \\ ${ }^{2}$ Yellow River Survey, Planning, Design and Research Institute Co., Ltd., Zhengzhou 450003, China \\ Correspondence should be addressed to Zhengyu Liu; liuzhengyu@sdu.edu.cn
}

Received 17 July 2020; Revised 27 September 2020; Accepted 20 October 2020; Published 31 October 2020

Academic Editor: Pitthaya Jamsawang

Copyright (c) 2020 Lichao Nie et al. This is an open access article distributed under the Creative Commons Attribution License, which permits unrestricted use, distribution, and reproduction in any medium, provided the original work is properly cited.

\begin{abstract}
The ahead geological prospecting of severely weathered surrounding rock in a tunnel is challenging due to complex geological conditions. Hence, the best approach is to use a variety of geophysical exploration methods. In this study, we have applied seismic, electrical resistivity (ER), and transient electromagnetic (TEM) methodologies for ahead prospecting of the highly weathered and complex surrounding rock at the diversion tunnel of the Hongyan River to Stone River Water Transfer Project in Shaanxi Province, China. The seismic method was employed to detect structural information for an area of $100 \mathrm{~m}$ from the front of the tunnel, the TEM method was used to obtain the resistivity information within $60 \mathrm{~m}$, and the ER method was conducted to obtain detailed resistivity information over a range of $30 \mathrm{~m}$. The integrated results generated a comprehensive interpretation of the geological body located within $100 \mathrm{~m}$ of the front of the tunnel. We divided this geological area into intact, severely weathered, and slightly weathered sections. Information on the water content of each section was also produced. The results of subsequent excavations are consistent with our results, proving the effectiveness and feasibility of a comprehensive approach for analyzing highly weathered and complex surrounding rock.
\end{abstract}

\section{Introduction}

Water resources are critical for the development of human society. However, various factors, such as climate and geography, can lead to the uneven distribution of water resources both temporally and spatially, which hinders development in areas where water is scarce $[1,2]$. To address the issue of uneven allocation of water resources, China has constructed numerous water transfer projects, such as the South-to-North Water Transfer Project. Among these projects, the diversion tunnel is a primary engineering component, connecting two different river basins to achieve complementarity in the allocation of water resources. While the tunnel is under construction, geological bodies (e.g., karsts, faults, fracture zones, etc.), which easily form waterconducting structures, can become a major threat to construction [3-6]. These structures may cause disasters, for example, water inrush and landslides in the tunnel, and threaten the safety of engineering personnel. To avoid disasters and take appropriate measures in advance, it is useful to determine the geological properties at the front of the tunnel. Geophysical detection constitutes a fast, low-cost, and effective method and has been widely used in advanced tunnel detection [5-8]. The seismic detection method is based on the propagation laws for elastic waves in geological bodies. It allows detection over long distances, is sensitive to lithological interfaces, and has been widely applied in oil and gas exploration $[9,10]$. In addition, this method has been successfully utilized in engineering geophysical prospecting, including in tunnel construction [11-14]. The TEM method provides another useful geophysical detection method. This determines the distribution of geological features in the 
detection area by observing the secondary magnetic field generated by pulsed magnetic field excitation. Its high sensitivity to low-resistance anomalies enables wide application of the TEM method in explorations for mineral and water resources [15-18]. The ER method detects the distribution of geological features by observing the electric field resulting from a constant current source and the electrical differences of the medium. This method is an effective tool for investigating the hydrogeological characteristics of subsurface materials [19-24]. In recent decades, this technique has also frequently been applied in ahead prospecting for tunnel construction $[25,26]$.

In practical applications, each method is useful with different types of objects; however, there are also limitations to each $[6,14,27-32]$. For example, the seismic method cannot provide information on whether a geological body contains water, and it is difficult to obtain the geological information related to a fault or fracture zone by using the electrical method. These factors make it challenging for a single detection method to provide a comprehensive understanding of geological bodies exhibiting complex features such as water-bearing fractures and water-bearing faults. Therefore, previous studies have obtained more detailed information on geological properties by combining various methods [33-35]. This combined approach constitutes a simple, fast, and effective method for comprehensively modeling a geological body. For example, Nie et al. combined seismic and ER methods to conduct comprehensive ahead prospecting for Metro Line R3 in Qingdao, China [36]. They reconstructed the distribution of an aquifer sand layer, and this comprehensive prospecting scheme played an important role in the construction of Metro tunnels. Many applications demonstrate that the interpretation of geological bodies realized by combining multiple geophysical exploration methods can lead to a more comprehensive understanding, and the results from different methods can effectively improve the accuracy of the interpretation.

The research in this study is related to the construction of a diversion tunnel of the "Hongyan River to Stone River" Water Transfer Project in Shaanxi Province, China. The project area and the water diversion tunnel are located in the hinterland of the Qinling Mountains. Several faults have developed in this area, and weathered rock masses, poor geological conditions, and the potential for floods pose threats to tunnel construction. Water inrush was observed when the tunnel was excavated to $\mathrm{K} 10+454$ and drilling results identified a water-bearing anomaly in front of the tunnel face. Therefore, comprehensive geological prospecting was conducted as it is necessary to determine the structure and water content of the geological body. The seismic method, TEM method, and ER method and the detection and data processing steps of these three methods were adopted in this exploration. Using the results obtained by the three methods, we interpret the geological body in front of the tunnel and divide the geological body into sections based on weathering degree and water content. Finally, excavation results provide data on rock conditions and water content, and this information is used to verify the effectiveness and feasibility of this comprehensive ahead prospecting approach.

\section{Study Area and Geological Environment}

The Hongyan River to Stone River Water Transfer Project is located in Taibai County, Shaanxi Province (Figure 1). This project is a component of the West Route Water Diversion plan for the South-to-North Water Diversion Project. The project is designed to connect the Hongyan River, a tributary of the Baohe River in the Yangtze River system, to the Taochuan River, a tributary of the Shitou River in the Yellow River system and provide an annual water transfer volume of 94 million $\mathrm{m}^{3}$. The water diversion tunnel passes through two geomorphic units of the Eroded Zhongshan and Taibai basin, from west to east. The study area is located in the middle section of the tunnel within the Taibai basin. The elevation ranges from 1463 to $1900 \mathrm{~m}$, the ground elevation of the tunnel axis range is 1690 to $1760 \mathrm{~m}$, and the tunnel line is approximately $8 \mathrm{~km}$ long $(\mathrm{K} 10+000$ to $\mathrm{k} 18+000 \mathrm{~m})$, buried at a depth of 220 to $300 \mathrm{~m}$. Detailed pregeological exploration determined the main stratum lithologies of the diversion tunnel to be the Middle Proterozoic Qinling Lower Subgroup (Pt2ql1), the Central Subgroup (Pt2ql2), the Upper Subgroup (Pt2q13), and a small amount of granite. The Middle Proterozoic Qinling Central Subgroup (Pt2q12) is distributed in the middle of the water diversion line, which is primarily composed of marble, hornblende schist, and gneiss. The water diversion tunnel is located in the hinterland of the Qinling Mountains. The longterm in situ stress has led to the formation of a basement structure dominated by east-west folds and faults. Remote sensing and field investigations indicate that 26 main fault structures exist within the project area. These structures are intensively developed and cut each other, lending considerable complexity. Additionally, there are many dense fracture zones and water-bearing areas associated with the fault. These unfavorable geological features all constitute potential threats to the tunnels.

The case area in this study is located in the middle of the tunnel at $\mathrm{K} 10+454 \mathrm{~m}$. The rock surrounding the tunnel is mainly marble. A nearby fault was identified during an early investigation (Figure 1). Due to the influence of the fault system, the surrounding rock has been severely weathered. When the side hole was excavated to $\mathrm{K} 10+454 \mathrm{~m}$, water inrush resulted at the tunnel face. The complexity of the current geological environment resulted in priority being given to the exploration of the geological environment in the front of the side tunnel and measures being taken to avoid possible disasters and ensure construction safety. The ahead drilling method was initially used and the results indicated the presence of anomalies in front of the working face. To obtain more detailed information on the geological properties, geophysical methods for comprehensive ahead prospecting were performed. 


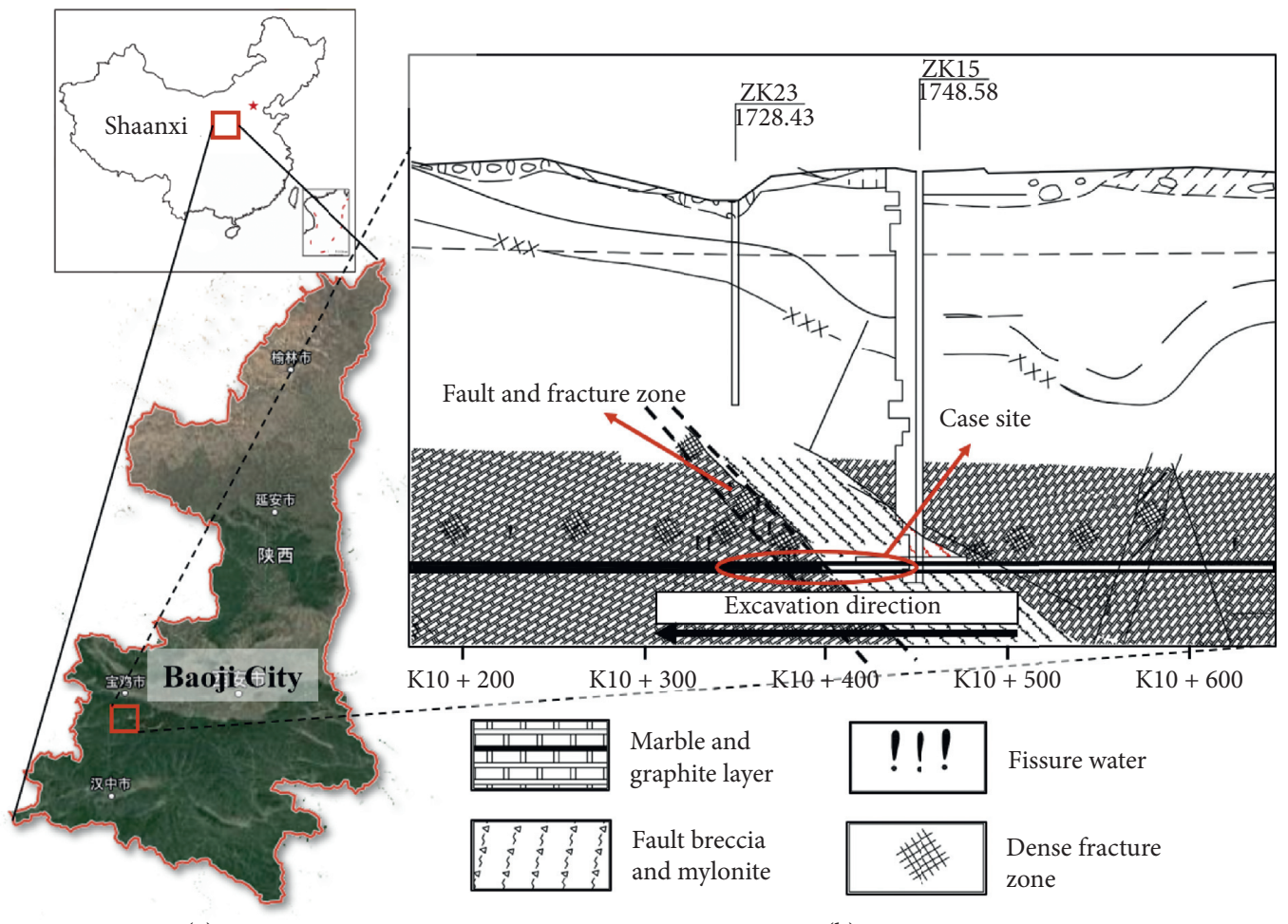

(a)

(b)

Figure 1: Schematic diagram of case area location. (a) Location of case project. (b) Geological section map for the case area location.

\section{Prospecting Method}

To ensure the safety of the project in this complex geological environment, it is necessary to obtain information on rock structure and water content in front of the tunnel. Seismic methods are sensitive to lithological interfaces, have long detection ranges, and can provide structural information on the rock mass in the front of the tunnel $[6,8]$. TEM and ER are electromagnetic methods that are sensitive to the presence of conductive media $[15,27,30]$. Therefore, these methods can obtain information on water bodies at short and medium distances in front of the tunnel. However, in this case, the utility of each method when applied individually may be significantly reduced. For example, due to the severe weathering of the rock mass, which has resulted in many reflective surfaces, the results of the seismic method may not be optimal. Therefore, we applied a comprehensive ahead prospecting process to attain detailed information on the geological environment. The process is outlined in Figure 2.

The entire process includes the application of three methods: the seismic method, the TEM method, and the ER method. The seismic method is applied to obtain structural information (e.g., faults, fractures, and karsts over a long range (approximately $100 \mathrm{~m}$ )). TEM can provide information on water-bearing structures over medium ranges (approximately $60 \mathrm{~m}$ ). Finally, the ER method can deliver detailed images of water-bearing structures over a short range (approximately $30 \mathrm{~m}$ ). The results of the three methods can be tested in the final interpretation step. For example, the results of the electromagnetic methods can verify the existence of water-containing structures detected by seismic methods. The results of the ER method can be utilized to provide thorough information for verification and supplementation of TEM close-range imaging results. This final process enables a comprehensive interpretation of the water-bearing structures.

\section{Seismic Method}

The three-dimensional (3D) seismic observation system proposed by Liu et al. has been applied in this study [37]. There are two excitation faces located within $4 \mathrm{~m}$ of the tunnel face (Figure 3). The distance between these three faces, together with the tunnel face, is $2 \mathrm{~m}$. Three shot points were distributed on each side of the excitation face, and the four receiving faces were arranged at $5 \mathrm{~m}$ intervals beginning at a point $10-20 \mathrm{~m}$ away from the tunnel face. Each receiving face is equipped with two geophones. A receiving face can be distinguished as one of two types according to the location of the geophones. In the first type, two geophones are located at the upper part of the receiving face, and the geophones in the second type are located at the lower part of the face. This observation system differs from the system proposed by Chen et al. due to the location of the shot point and the geophones [34]. The surrounding rock conditions are unsatisfactory; hence, some places are not suitable for geophone installation.

A hammer source was used in this study. While hammering the rock body to generate seismic waves, the trigger signal will be generated and transmitted to the base station. The base station sends the geophones a command to collect seismic waves and transmits the seismic wave data returned 
Comprehensive ahead prospecting method

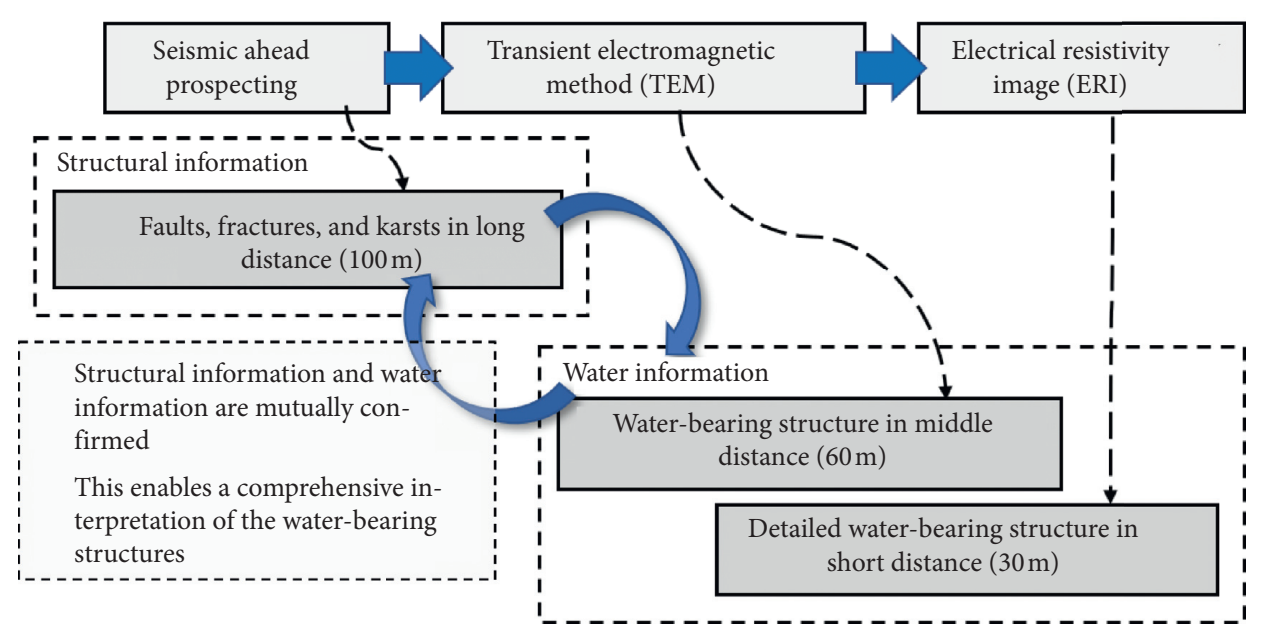

Figure 2: Process for comprehensive ahead prospecting.
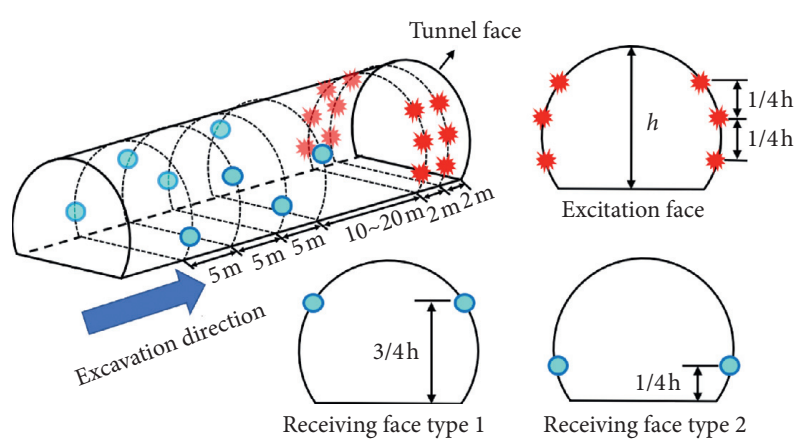

Shot points

Geophones

FIGURE 3: Schematic of instrument arrangement and observation system for seismic method.

by the geophones to the laptop. The seismic data collected by geophones will be processed by our self-written data processing code based on the "Kirchhoff prestack depth migration" (KPSDM) method for 3D imaging [38, 39].

\section{TEM}

The TEM observation system applied in this study is depicted in Figure 4. The central loop device was arranged on the tunnel face; the length of the transmitting coil was $2 \mathrm{~m}$, and the number of turns was 56. The current in the transmitting coil was $1 \mathrm{~A}$, and the transmitting frequency was $6.25 \mathrm{~Hz}$. The effective area of the receiving coil was $31.4 \mathrm{~m}^{2}$. The ProTEM47HP transient electromagnetic instrument (Geonics, Canada) was used for data acquisition and processing [40]. The TEM measuring line was arranged on the tunnel face and consisted of several measurement points. The initial measurement point was $1 \mathrm{~m}$ from the left sidewall of the tunnel. The distance between the measurement points was $0.2 \mathrm{~m}$. When measuring, the center of the coil device was located at the measuring point. After completing this measurement, it was moved to the right to the next measuring point in order to continue the measurement process. The detected data were then transmitted to the host and ultimately processed by the supplementary data processing software into an apparent resistivity image.

\section{ER Method}

The ER method observation system applied in the 2017 study conducted by Bu et al. $[26,41]$ was employed in the present study (Figure 5). However, in contrast to Bu et al., the DC resistivity method was applied in the current case. The spacing between the current electrode loops was fixed $(2 \mathrm{~m})$. Two measuring lines were arranged on the tunnel face, and eight potential electrodes were arranged on each measuring line. During the measurement, the current was first injected by the current electrode on $\mathrm{A} 1$ and the potential values were measured with the 16 potential electrodes. The current was then injected by A2-An sequentially, and the corresponding potential values were measured to complete the entire detection process. The data were processed into a $3 \mathrm{D}$ resistivity image of the geological properties in front of the tunnel by our self-written 3D ER inversion code based on the smooth constrained least-squares optimization method [30].

\section{Results and Discussion}

7.1. Seismic Method Results. The results of the seismic method are shown in Figure 6, and the wave velocity range information for each section is outlined in Table 1. The data demonstrate the presence of two large reflection areas in front of the tunnel. In the section directly in front of the tunnel face $(0$ to $20 \mathrm{~m})$, there is almost no reflection, and average wave velocity in this section is high. Therefore, it is speculated that the surrounding rocks in this section may be integrated. A strong reflection area exists in an area 20 to $60 \mathrm{~m}$ (approximately between $\mathrm{K} 10+434 \mathrm{~m}$ and $\mathrm{K} 10+394 \mathrm{~m}$ ) from the tunnel face, and the wave velocity in 


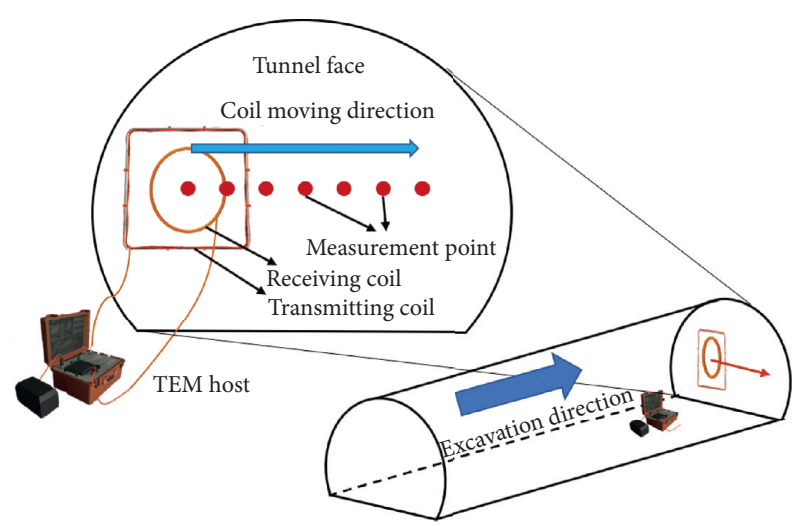

FIgURE 4: Schematic of instrument arrangement and observation system for TEM.

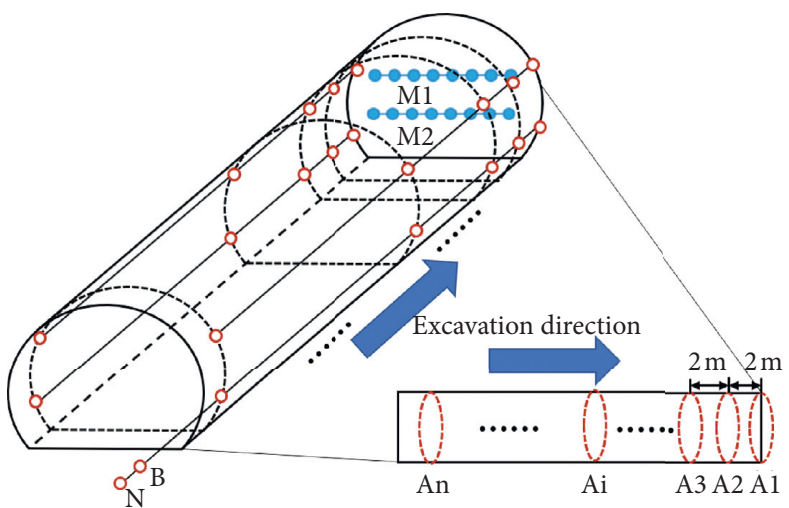

- Current electrode

- Measuring electrode

Figure 5: Schematic of the ER method observation system.

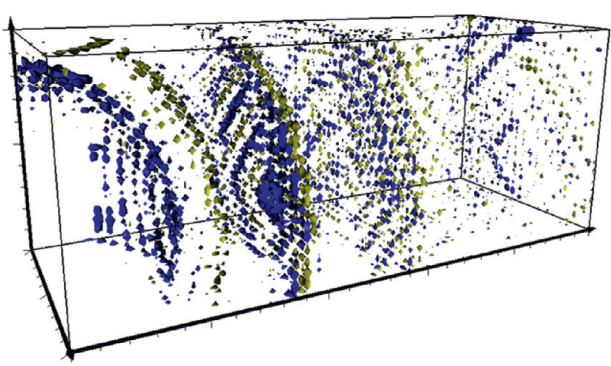

(a)

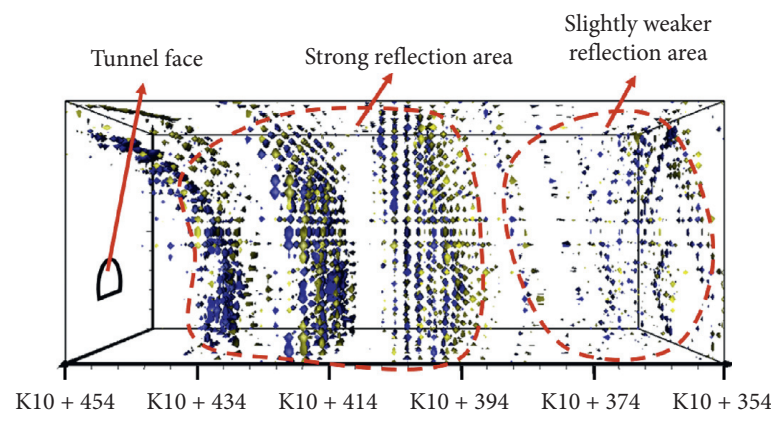

(b)

FIGURE 6: 3D image of seismic wave reflection scanning imaging. (a) General view of seismic reflection imaging. (b) Side view of seismic reflection imaging.

this section is low. This infers severe weathering and fragmentation of rocks in this area, which also suggests the likelihood of this as a water-rich area. The other reflection area is located 60 to $100 \mathrm{~m}$ (approximately between $\mathrm{K} 10+394 \mathrm{~m}$ and $\mathrm{K} 10+354 \mathrm{~m}$ ) from the tunnel face. The reflection in this area is slightly weaker than that in the previous area, and the wave velocity is also slightly higher than that in the previous section. There are two main reflection bands in this area, located on both sides of the tunnel advancing axis; there may be weathering of the rock here. However, due to lacking additional water content condition information, it is unknown whether it will contain water and directly affect the tunnel when it is driven into the area. 
TABLE 1: Wave velocity range in each section.

\begin{tabular}{lr}
\hline Mileage range & Wave velocity range (m/s) \\
\hline $\mathrm{K} 10+454-\mathrm{K} 10+434$ & $2891-3000$ \\
$\mathrm{~K} 10+434-\mathrm{K} 10+404$ & $2005-2132$ \\
$\mathrm{~K} 10+394-\mathrm{K} 10+358$ & $2223-2399$ \\
\hline
\end{tabular}

7.2. TEM Results. The TEM results are presented in Figure 7 and show the distribution of apparent resistivity in an area within $60 \mathrm{~m}$ of the front of the tunnel. A high-resistance area is located within $20 \mathrm{~m}$ of the tunnel surface and is primarily distributed on the right side of the tunnel. A small portion of the high-resistance area extends forward to a point $40 \mathrm{~m}$ from the tunnel face. The low-resistance area is mainly distributed on the right side of the space in an area ranging $0-20 \mathrm{~m}$ from the tunnel face and is observed in most areas in the range of $20-60 \mathrm{~m}$ from the tunnel face. However, the accuracy of the TEM process is limited by the presence of various electrical components, such as lighting near the tunnel face and lighting cables, and by holes that interfere with transient electromagnetic data. Regardless, the hydrogeology in the front of the tunnel can still be analyzed from the results:

(1) The area from mileage $\mathrm{K} 10+454 \mathrm{~m}$ to $\mathrm{K} 10+434 \mathrm{~m}$ has a relatively high apparent resistivity on the right side, while the resistivity on the left is relatively low. It can be inferred that the rocks in this area are largely intact, and those on the left may contain water.

(2) The apparent resistivity of the area after the mileage $\mathrm{K} 10+434$ is relatively low. It is inferred that the rock mass in this area is rich in water.

7.3. ER Method Results. Figure 8 shows the 3D inversion results obtained from the ER method. The $X$ direction is the vertical direction, the $Y$ direction represents the width direction of the face, and the $Z$ direction indicates the excavation direction. The coordinate origin is at the center of the tunnel face. The results indicate that the area near the tunnel face (approximately in the range of $0-20 \mathrm{~m}$ ) is primarily a high-resistance area, while there is a small low-resistance area on the left side of the tunnel face (Figure 8). The primary low-resistance area is distributed within a range of 15-30 m from the tunnel face. The slice images of the inversion results are shown in Figure 8 and provide more detailed resistivity information. The range of the high-resistivity area is seen to be shrinking along the negative direction of the $x$-axis. From these results, we can infer the following:

(1) The resistivity is higher in the range of 0-15 $\mathrm{m}$ from the front of the tunnel face, and the rock may be relatively intact. There is a small low-resistance area on the front left side of the tunnel face, which may contain water.

(2) There is a sizeable low-resistance area located beyond the point $15 \mathrm{~m}$ from the tunnel face. The rock mass in this area is likely to be rich in water.
7.4. Interpretation and Discussion. The comprehensive interpretation of geological properties is based on the data from the three methods. The geological properties located within $100 \mathrm{~m}$ of the front of the tunnel can be divided into three sections (Figure 9). The first section ranges from 0 to $20 \mathrm{~m}$ (mileage $\mathrm{K} 10+454 \mathrm{~m}-\mathrm{K} 10+434 \mathrm{~m}$ ) from the tunnel and is marked by celeste. The results of the seismic method demonstrate that the wave velocity through the medium in this section is high, and there are few reflective interfaces in this section. The results of the TEM and the ER methods show that this is a high-resistance section, and there is a small low-resistivity area on the left side of the face. We can infer that the rock in this section exhibits good integrity, and the overall water content of the rock is low. The second section is located between 20 and $60 \mathrm{~m}$ (mileage $\mathrm{K} 10+434 \mathrm{~m}-\mathrm{K} 10+394 \mathrm{~m}$ ) from the tunnel face. The seismic method shows that the rock condition in this section is extremely poor, and the rock is severely weathered. The TEM and ER methods indicate that the resistivity in this section is low, and there may be abundant water in the rock mass. Therefore, we speculate that this area may be a watercontaining fracture zone. Excavation could cause disasters, including water inrush and tunnel collapse, and it is necessary to adopt precautionary measures. The remainder of Figure 9 depicts the third section. The seismic method demonstrates that rock in this section is slightly weathered. Two fracture zones may exist in this section and these would adversely affect the safety of the tunnel. However, due to the limitations of detection distance, the results of the TEM and ER methods cannot provide water content condition information for the interpretation of the geological properties. Nonetheless, preventive measures should also be adopted during construction in this area.

Excavation photographs are shown in Figure 9 and these confirm that the rock on the tunnel face and the sidewall are intact (Figure 9), with almost no water inrush from the tunnel face. This corresponds with the prediction of geological conditions. When the tunnel was excavated into the second section, the condition of the rock mass in the front of the tunnel deteriorated sharply. The surrounding rocks are severely weathered, and block dropping occurs from the top wall of the tunnel. Considerable water inrush also occurred from the tunnel face. The overall condition of the rock mass in this section is consistent with the predicted results. After providing prereinforcement and improving the pumping and drainage capacity of the tunnel, the tunnel excavation process passed this section safely. The rock in the third section is slightly weathered, and water inrush still occurs. This is consistent with the situation predicted by the seismic method results. By comparing and analyzing the results of the subsequent excavation, we can conclude that the comprehensive ahead prospecting predicted the geological 


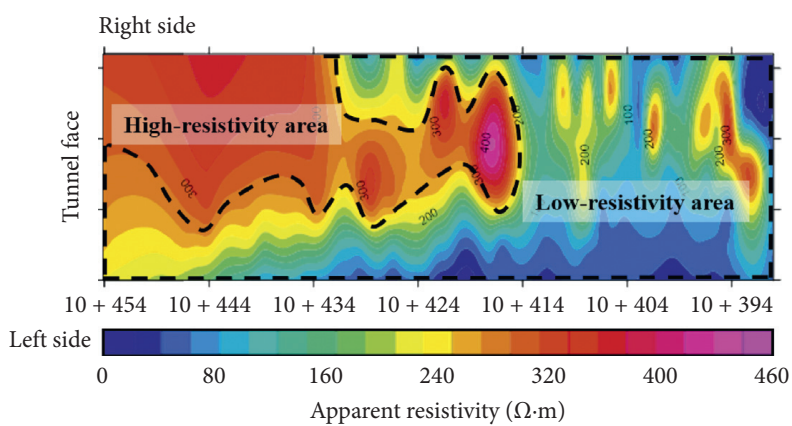

FIGURE 7: Apparent resistivity contour image from TEM.

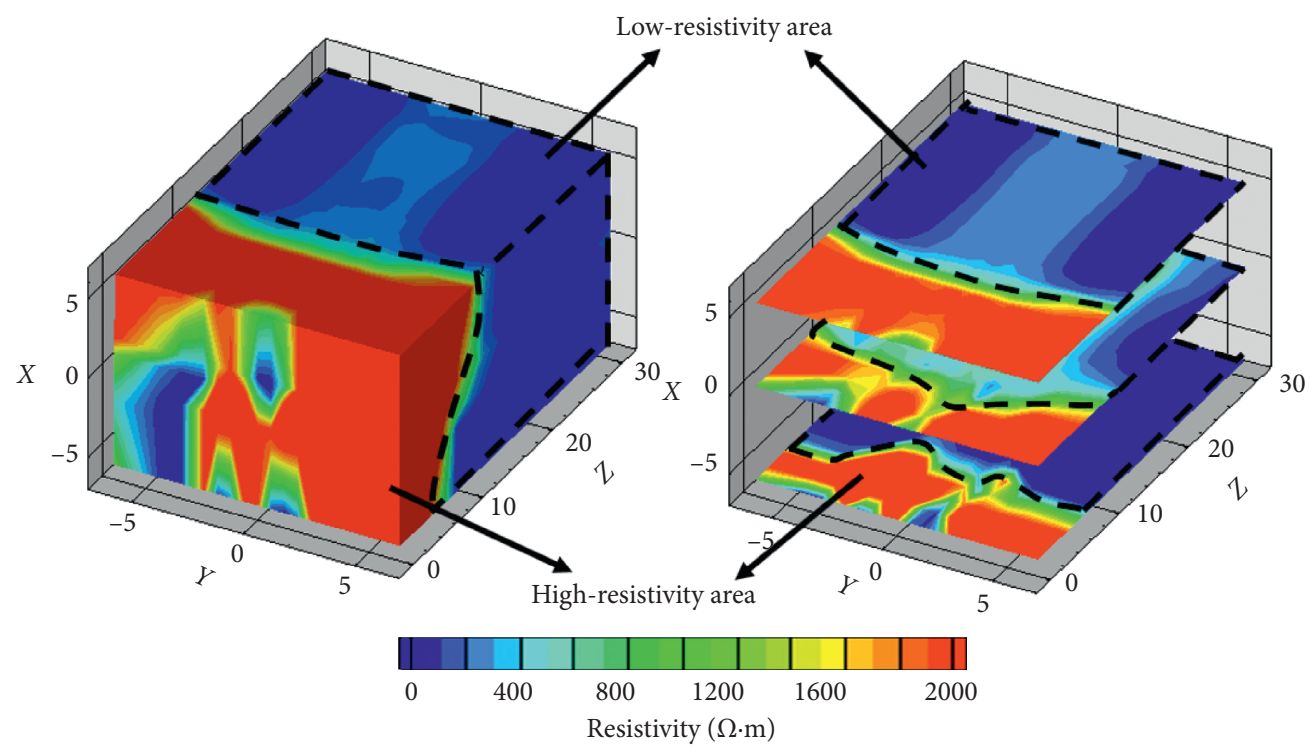

FIGURE 8: 3D inversion resistivity imaging results. (a) 3D image of resistivity distribution. (b) $2 \mathrm{D}$ slice resistivity image in three planes.

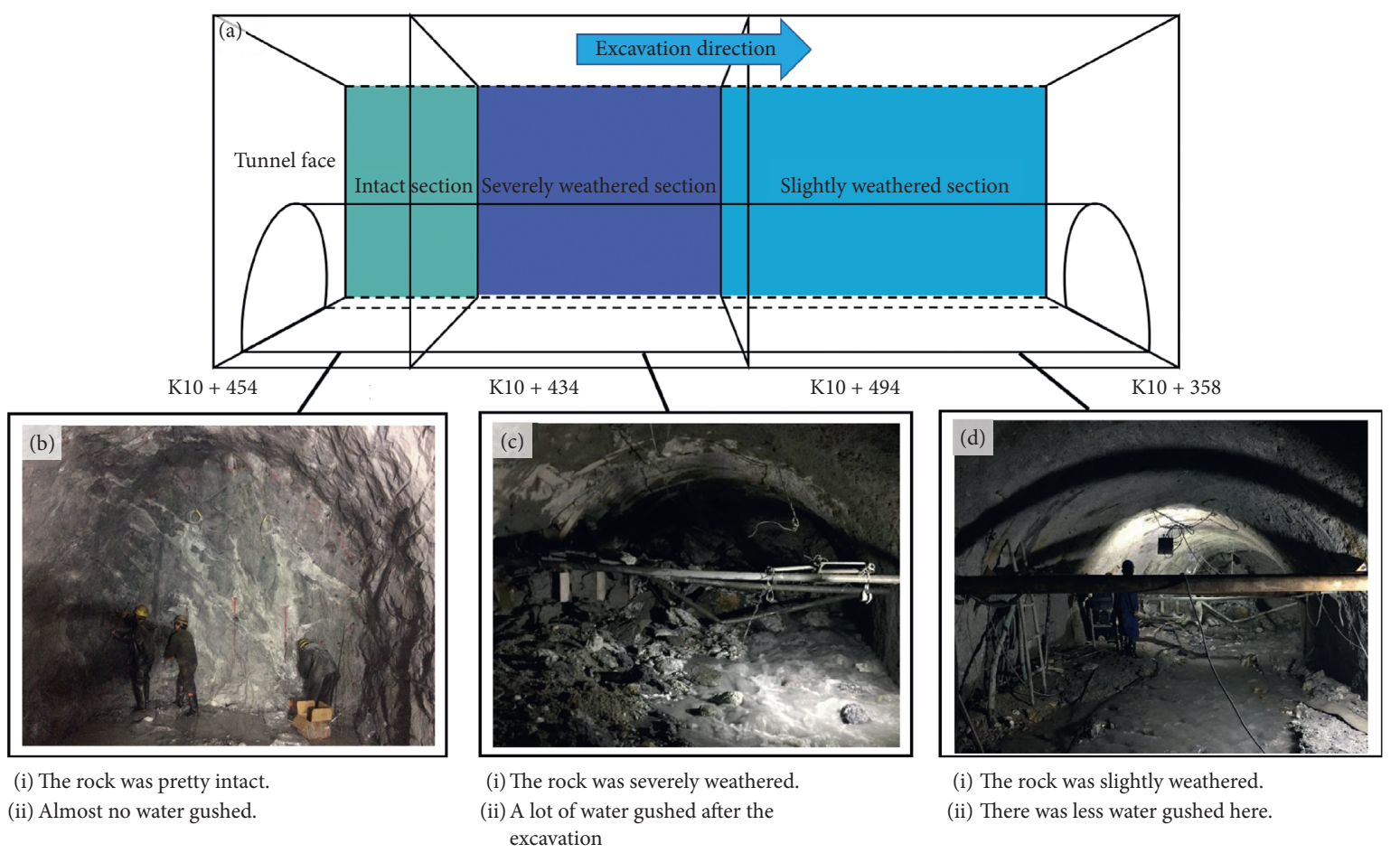

Figure 9: Comprehensive interpretations and excavation photos for each section. (a) Comprehensive map of rock masses within $100 \mathrm{~m}$ of the front of the tunnel. (b) Excavation photo of the first section. (c) Excavation photo of the second section, with considerable water pouring. (d) Excavation photo of the third section, where water flow still occurred. 
properties in front of the tunnel. This allowed construction personnel to take reasonable precautions to avoid possible disaster and allow the tunnel to pass safely through this extremely weathered water-bearing fracture zone.

\section{Conclusions}

In this study, the geological prospecting case of a severely weathered rock mass environment was investigated. According to the results of a previous geological survey and advanced drilling, a possibility of a large-scale water-bearing fracture zone in front of the case site was established. In this complex geological environment, the application of a single geophysical exploration method has limitations. It is difficult to accurately determine the geological structure and occurrence of water in front of the tunnel. To achieve reasonable predictions of geological properties, a comprehensive ahead prospecting and interpretation method has been applied. This includes the use of the seismic method, ER method, and the TEM method. From the results, the following preliminary conclusions can be drawn:

(1) From the seismic method, it is concluded that there may be two weathered areas in front of the tunnel. The rocks located in the range of $0-20 \mathrm{~m}$ from the front of the tunnel are relatively intact, with a low degree of weathering. The surrounding rock located within $20-50 \mathrm{~m}$ is extremely fractured; it may be severely weathered rock, and it is highly likely that it contains water. The surrounding rock in the range of 60-100 m may be slightly broken and may contain water.

(2) The results of the TEM method indicate the resistivity distribution for the area located $0-60 \mathrm{~m}$ in front of the tunnel. The results for the area located $0-20 \mathrm{~m}$ from the face of the tunnel show that this area has relatively high resistance, and there is a low probability of it containing water. The area located in $20-60 \mathrm{~m}$ is a low-resistivity area, and there is a high probability of water content. However, due to the existence of electromagnetic interference and measurement error, the results of the TEM method may still exhibit problems (e.g., inaccuracy and false anomalies), so the resistivity distribution requires further exploration and verification.

(3) The results of the ER method provide increasingly accurate resistivity information for the area located $0-30 \mathrm{~m}$ in front of the tunnel. The results indicate that the high-resistance area is in the range of $0-15 \mathrm{~m}$, and the remaining area exhibits low resistance.

Based on the results from the three methods, we have undertaken a comprehensive geological evaluation and divided the geological body located within $100 \mathrm{~m}$ from the front of the tunnel into three sections. The first section extends $0-20 \mathrm{~m}$ from the face of the tunnel. The seismic method results and resistivity information both show that the rocks in this section are largely intact, and the probability that they contain water is low. The second section is located 20-60 $\mathrm{m}$ from the face of the tunnel. The surrounding rock in this section is severely weathered and extremely broken, and the low resistivity indicates that the section may be rich in water. The third section is located $60-100 \mathrm{~m}$ from the face of the tunnel and the surrounding rock in this section may be slightly weathered. The results of a subsequent excavation confirm the results of the comprehensive interpretation. The comprehensive geophysical method is therefore effective and can feasibly be applied to study the highly weathered and complex geological environments faced in tunnel construction. However, if the environment is extremely complex and it is vital to know the water content information of all sections, continuous ER and TEM detection will be necessary in order to obtain detailed information. This will significantly increase the cost of detection. Some additional information such as rock sample information obtained from advanced borehole could be used for helping comprehensive interpretation. Furthermore, some joint inversion methods such as cross-gradient inversion and fuzzy $c$-means clustering inversion can significantly improve the effect of imaging and are worthy of being applied to the comprehensive ahead prospecting in tunnels. In the future of tunnel comprehensive detection, in addition to various inversion methods, various detection methods are also worthy of consideration, such as GPR and even flow field inversion.

\section{Data Availability}

The data used to support the findings of this study are available from the corresponding author upon request.

\section{Conflicts of Interest}

The authors declare no conflicts of interest.

\section{References}

[1] Y. Yang, L. Yin, and Q. Zhang, "Quantity versus quality in China's South-to-North water diversion project: a system dynamics analysis," Water, vol. 7, no. 12, pp. 2142-2160, 2015.

[2] H. Fu and X. Yang, "Effects of the South-North water diversion project on the water dispatching pattern and ecological environment in the water receiving area: a case study of the fuyang river basin in handan, China," Water, vol. 11, no. 4, p. 845, 2019.

[3] L. Li, T. Lei, S. Li et al., "Risk assessment of water inrush in karst tunnels and software development," Arabian Journal of Geosciences, vol. 8, no. 4, pp. 1843-1854, 2015.

[4] D.-x. Liang, Z.-q. Jiang, S.-y. Zhu, Q. Sun, and Z.-w. Qian, "Experimental research on water inrush in tunnel construction," Natural Hazards, vol. 81, no. 1, pp. 467-480, 2016.

[5] S. Li, B. Liu, L. Nie et al., "Detecting and monitoring of water inrush in tunnels and coal mines using direct current resistivity method: a review," Journal of Rock Mechanics and Geotechnical Engineering, vol. 7, no. 4, pp. 469-478, 2015.

[6] S. Li, B. Liu, X. Xu et al., "An overview of ahead geological prospecting in tunneling," Tunnelling and Underground Space Technology, vol. 63, pp. 69-94, 2017.

[7] S. A. S. Araffa, H. S. Sabet, and W. R. Gaweish, "Integrated geophysical interpretation for delineating the structural 
elements and groundwater aquifers at central part of Sinai Peninsula, Egypt," Journal of African Earth Sciences, vol. 105, pp. 93-106, 2015.

[8] S. C. Li, Z. Q. Zhou, Z. H. Ye, L. P. Li, Q. Q. Zhang, and Z. H. Xu, "Comprehensive geophysical prediction and treatment measures of karst caves in deep buried tunnel," Journal of Applied Geophysics, vol. 116, pp. 247-257, 2015.

[9] Y. Ma, X. Guo, T. Guo, R. Huang, X. Cai, and G. Li, "The puguang gas field: new giant discovery in the mature sichuan basin, southwest China," Aapg Bulletin, vol. 91, no. 5, pp. 627-643, 2007.

[10] C. Jia, M. Zheng, and Y. Zhang, "Unconventional hydrocarbon resources in China and the prospect of exploration and development," Petroleum Exploration and Development, vol. 39, no. 2, pp. 139-146, 2012.

[11] D. Marti, R. Carbonell, I. Flecha et al., "High-resolution seismic characterization in an urban area: subway tunnel construction in Barcelona, Spain," Geophysics, vol. 73, no. 2, 2008.

[12] L. Petronio and F. Poletto, "Seismic-while-drilling by using tunnel boring machine noise," Geophysics, vol. 67, no. 6, pp. 1798-1809, 2002.

[13] F. Poletto and L. Petronio, "Seismic interferometry with a TBM source of transmitted and reflected waves," Geophysics, vol. 71, no. 4, 2006.

[14] A. Alimoradi, A. Moradzadeh, R. Naderi, M. Z. Salehi, and A. Etemadi, "Prediction of geological hazardous zones in front of a tunnel face using TSP-203 and artificial neural networks," Tunnelling and Underground Space Technology, vol. 23, no. 6, pp. 711-717, 2008.

[15] H. Sun, X. Li, S. Li, Z. Qi, M. Su, and Y. Xue, "Multi-component and multi-array TEM detection in karst tunnels," Journal of Geophysics and Engineering, vol. 9, no. 4, pp. 359-373, 2012.

[16] S. Li, H. Sun, X. Lu, and X. Li, "Three-dimensional modeling of transient electromagnetic responses of water-bearing structures in front of a tunnel face," Journal of Environmental \& Engineering Geophysics, vol. 19, no. 1, pp. 13-32, 2014.

[17] G. Xue, W. Chen, Z. Ma, and D. Hou, "Identifying deep saturated coal bed zones in China through the use of large loop tem," Journal of Environmental and Engineering Geophysics, vol. 23, no. 1, pp. 135-142, 2018.

[18] N. Zhou, G. Xue, D.-y. Hou, and Y. Lu, "An investigation of the effect of source geometry on grounded-wire tem surveying with horizontal electric field," Journal of Environmental and Engineering Geophysics, vol. 23, no. 1, pp. 143-151, 2018.

[19] Q. Gao, Y. Shang, M. Hasan, W. Jin, and P. Yang, "Evaluation of a weathered rock aquifer using ERT method in South guangdong, China," Water, vol. 10, no. 3, p. 293, 2018.

[20] G. Raffelli, M. Previati, D. Canone et al., "Local- and plot-scale measurements of soil moisture: time and spatially resolved field techniques in plain, hill and mountain sites," Water, vol. 9, no. 9, p. 706, 2017.

[21] C. Masciopinto, I. Liso, M. Caputo, and L. De Carlo, “An integrated approach based on numerical modelling and geophysical survey to map groundwater salinity in fractured coastal aquifers," Water, vol. 9, no. 11, p. 875, 2017.

[22] P. B. Wilkinson, J. E. Chambers, P. I. Meldrum, R. D. Ogilvy, and S. Caunt, "Optimization of array configurations and panel combinations for the detection and imaging of abandoned mineshafts using 3D cross-hole electrical resistivity tomography," Journal of Environmental \& Engineering Geophysics, vol. 11, no. 3, pp. 213-221, 2006.
[23] B. Liu, Q. Guo, S. Li et al., "Deep learning inversion of electrical resistivity data," IEEE Transactions on Geoscience and Remote Sensing, pp. 1-14, 2020.

[24] B. Liu, Y. Pang, D. Mao et al., "A rapid four-dimensional resistivity data inversion method using temporal segmentation," Geophysical Journal International, vol. 221, no. 1, pp. 586-602, 2020.

[25] B.-Y. Ruan, X.-K. Deng, H.-F. Liu, L. Zhou, and L. Zhang, "Research on a new method of advanced focus detection with DC resistivity in tunnels," Chinese Journal of Geophysics, vol. 52, no. 1, pp. 250-259, 2009.

[26] L. Shu-Cai, N. Li-Chao, B. Liu, and M.-Z. Tian, "Advanced detection and physical model test based on multi-electrode sources array resistivity method in tunnel," Chinese Journal of Geophysics-Chinese Edition, vol. 58, no. 4, pp. 1434-1446, 2015.

[27] A. Revil, M. Karaoulis, T. Johnson, and A. Kemna, "Review: some low-frequency electrical methods for subsurface characterization and monitoring in hydrogeology," Hydrogeology Journal, vol. 20, no. 4, pp. 617-658, 2012.

[28] E. Auken, L. Pellerin, N. B Christensen, and K. Sørensen, “A survey of current trends in near-surface electrical and electromagnetic methods," Geophysics, vol. 71, no. 5, 2006.

[29] J. Peacock and A. King, "Central loop transient electromagnetic soundings," Exploration Geophysics, vol. 16, no. 2-3, pp. 261-265, 1985.

[30] M. H. Loke, J. E. Chambers, D. F. Rucker, O. Kuras, and P. B. Wilkinson, "Recent developments in the direct-current geoelectrical imaging method," Journal of Applied Geophysics, vol. 95, pp. 135-156, 2013.

[31] A. Kemna, A. Binley, G. Cassiani et al., "An overview of the spectral induced polarization method for near-surface applications," Near Surface Geophysics, vol. 10, no. 6, pp. 453-468, 2012.

[32] Y.-j. Xue, J.-x. Cao, X.-j. Wang, Y.-x. Li, and J. Du, "Recent developments in local wave decomposition methods for understanding seismic data: application to seismic interpretation," Surveys in Geophysics, vol. 40, no. 5, pp. 1185-1210, 2019.

[33] W. Zheng, X. Li, N. Lam et al., "Applications of integrated geophysical method in archaeological surveys of the ancient Shu ruins," Journal of Archaeological Science, vol. 40, no. 1, pp. 166-175, 2013.

[34] L. Chen, F. Zhang, R. Yuxiao, X. Xinji, Y. Zhichao, and L. Ming, "Tunnel prospecting based on integrated interpretation of geophysical data: Xiangyun tunnel, Yunnan province, China," Journal of Environmental and Engineering Geophysics, vol. 24, pp. 63-75, 2019.

[35] B. Liu, Q. Guo, Z. Liu et al., "Comprehensive ahead prospecting for hard rock TBM tunneling in complex limestone geology: a case study in Jilin, China," Tunnelling and Underground Space Technology, vol. 93, Article ID 103045, 2019.

[36] L. Nie, Z. Ma, C. Wang et al., "Integrated ERT, seismic, and electrical resistivity imaging for geological prospecting on Metro line R3 in Qingdao, China," Journal of Environmental and Engineering Geophysics, vol. 24, no. 4, pp. 537-547, 2019.

[37] B. Liu, L. Chen, S. Li et al., "A new 3D observation system designed for a seismic ahead prospecting method in tunneling," Bulletin of Engineering Geology and the Environment, vol. 77, no. 4, pp. 1547-1565, 2018.

[38] Y. Ashida, "Seismic imaging ahead of a tunnel face with threecomponent geophones," International Journal of Rock Mechanics and Mining Sciences, vol. 38, no. 6, pp. 823-831, 2001. 
[39] J. Tzavaras, S. Buske, K. Groß, and S. Shapiro, "Three-dimensional seismic imaging of tunnels," International Journal of Rock Mechanics and Mining Sciences, vol. 49, pp. 12-20, 2012.

[40] J. L. Porsani, C. A. Bortolozo, E. R. Almeida, E. N. S. Sobrinho, and T. G. d. Santos, "TDEM survey in urban environmental for hydrogeological study at USP campus in São Paulo city, Brazil," Journal of Applied Geophysics, vol. 76, pp. 102-108, 2012.

[41] L. Bu, S. Li, S. Shi et al., "Application of the comprehensive forecast system for water-bearing structures in a karst tunnel: a case study," Bulletin of Engineering Geology and the Environment, vol. 78, no. 1, pp. 357-373, 2019. 